Marc A. Meier, Manuel Ottiger, Alaadin Vögeli, Christian Steuer, Luca Bernasconi, Robert Thomann, Mirjam Christ-Crain, Christoph Henzen, Claus Hoess, Werner Zimmerli, Andreas Huber, Beat Mueller and Philipp Schuetz*

\title{
Activation of the tryptophan/serotonin pathway is associated with severity and predicts outcomes in pneumonia: results of a long-term cohort study
}

DOI 10.1515/cclm-2016-0912

Received October 11, 2016; accepted November 1, 2016; previously published online January 11, 2017

\section{Abstract}

Background: As part of the immune defense during infection, an increase in enzyme activity of indoleamine 2,3-dioxygenase (IDO) leads to a breakdown of tryptophan to kynurenine. In previous animal studies, therapeutic antagonism of IDO resulted in reduced sepsis mortality. We investigated the prognostic ability of tryptophan, serotonin, kynurenine and IDO (represented by the ratio of kynurenine/tryptophan) to predict adverse clinical outcomes in patients with community-acquired pneumonia (CAP).

Methods: We measured tryptophan, serotonin and kynurenine on admission plasma samples from CAP patients included in a previous multicenter trial by liquid chromatography coupled with tandem mass spectrometry (LC-MS/MS). We studied their association with inflammation (C-reactive protein), infection (procalcitonin) and clinical outcome.

Results: Mortality in the 268 included patients was $45 \%$ within 6 years of follow-up. IDO and kynurenine showed a

\footnotetext{
*Corresponding author: Prof. Dr. med. Philipp Schuetz, MD, MPH, University Department of Medicine, Kantonsspital Aarau, Tellstrasse, CH-5001 Aarau, Switzerland, Phone: 00416283868 12, Fax: 00416283898 73, E-mail: schuetzph@gmail.com

Marc A. Meier, Manuel Ottiger, Alaadin Vögeli and Beat Mueller: Medical University Department, Kantonsspital Aarau, Aarau, Switzerland

Christian Steuer, Luca Bernasconi and Andreas Huber: Department of Laboratory Medicine, Kantonsspital Aarau, Aarau, Switzerland Robert Thomann: Medical Department, Buergerspital Solothurn, Solothurn, Switzerland

Mirjam Christ-Crain: Medical University Department, University Hospital Basel, Basel, Switzerland

Christoph Henzen: Medical Department, Kantonsspital Luzern, Luzern, Switzerland

Claus Hoess: Medical Department, Kantonsspital Muensterlingen, Muensterlingen, Switzerland

Werner Zimmerli: Medical University Department, Kantonsspital Baselland, Liestal, Switzerland
}

strong positive correlation with markers of infection (procalcitonin) and inflammation (C-reactive protein) as well as sepsis and CAP severity scores. Tryptophan showed similar, but negative correlations. In a multivariate regression analysis adjusted for age and comorbidities, higher IDO activity and lower tryptophan levels were strongly associated with short-term adverse outcome defined as death and/or ICU admission within 30 days with adjusted odds ratios of 9.1 [95\% confidence interval (CI) 1.4-59.5, $\mathrm{p}=0.021]$ and $0.11(95 \%$ CI $0.02-0.70, \mathrm{p}=0.021)$. Multivariate analysis did not reveal significant associations for kynurenine and serotonin.

Conclusions: In hospitalized CAP patients, higher IDO activity and lower tryptophan levels independently predicted disease severity and short-term adverse outcome. Whether therapeutic modulation of IDO has positive effects on outcome needs further investigation.

Keywords: community-acquired pneumonia; indoleamine 2,3-dioxygenase; kynurenine; long-term; mortality; prediction; serotonin; tryptophan.

\section{Introduction}

Tryptophan (TRP) is an essential amino acid and plays a key role in mammals as a precursor of physiological substances including serotonin 5-Hydroxytryptamine (5-HT) and melatonin [1]. Particularly TRP and its metabolites are thought to be altered during infections as part of the immune reaction $[2,3]$. There are two metabolic pathways in the breakdown of TRP [4] (illustrated in Figure 1). About $95 \%$ of TRP is metabolized via the kynurenine (KYN) pathway. The rate-limiting enzymes are tryptophan 2,3-dioxygenase (TDO) localized in hepatic tissue and indoleamine 2,3-dioxygenase (IDO) in extrahepatic tissue. IDO is inducible by interferon- $\gamma$ and is thus the rate limiting enzyme in an infective condition $[5,6]$. The serotonin 5-HT pathway is catalyzed by tryptophan hydroxylase (TPH), whereas less than $5 \%$ of TRP is metabolized by this pathway [4]. 


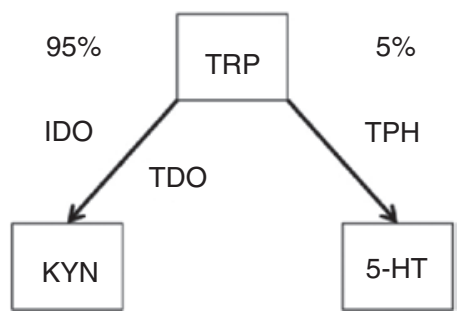

Figure 1: Tryptophan/serotonin pathway. TRP, tryptophan; KYN, kynurenine; IDO, indoleamine 2,3-dioxygenase; TDO, tryptophan 2,3-dioxygenase; $5-\mathrm{HT}$, serotonin; TPH, tryptophan hydroxylase.

Initially, IDO was recognized as a potent antimicrobial effector molecule. Its antimicrobial effect was thought to exist through a breakdown of TRP as TRP is essential for the growth of microorganisms. However, recent studies have demonstrated its novel role as a strong inhibitor of $\mathrm{T}$ cell proliferation [2]. Sepsis-induced elevation of 5-HT has recently been discovered in a mouse model where high levels of 5-HT facilitated endothelial hyperpermeability [3]. Microvascular dysfunction is believed to contribute to patient mortality in sepsis [7]. Therefore an intervention with a 5-HT transporter inhibitor was speculated to be a potential therapeutic option [3].

Community-acquired pneumonia (CAP) is currently the leading cause of death due to an infectious disease [8] and responsible for high costs, morbidity, and mortality worldwide $[9,10]$. Serum activity of IDO predicted shortterm prognosis in a small cohort of 129 CAP patients [11]. However, this study did not measure serotonin. In a state of infection, the catabolism of TRP is activated [12]. Given these results from mostly animal studies, there is interest in a larger clinical study investigating how adaptations in the TRP/5-HT pathway interact with severity and outcome in patients with a well-defined infection. This will allow better understanding whether therapeutic interventions targeted at this pathway could bring benefits.

Herein, our aim was to investigate the association of metabolites of the TRP/5-HT pathway with severity and outcome in a well-characterized cohort of patients hospitalized for CAP with a follow-up over more than six years.

\section{Materials and methods}

\section{Study design}

A total of 268 patients hospitalized for CAP and available blood specimens from a previous Swiss multicenter trial [13, 14] were included in this analysis. The initial trial included 925 (68\%) CAP patients in which all-cause mortality was $45 \%$ over a follow-up period of 6 years [15].

The initial trial was a randomized controlled, antibiotic stewardship trial performed between October 2006 and March 2008 at six Swiss secondary and tertiary care centers and described elsewhere [13]. The primary goal of the study was to verify the efficacy and safety of using the procalcitonin (PCT) level to guide the initiation and duration of antibiotic therapy in patients with lower respiratory tract infections [13, 14]. Patients at least 18 years of age presenting from the community or a nursing home to the emergency department (ED) were included if they fulfilled at least one of the following criteria: cough, dyspnea, pleural pain, sputum production or tachypnea and one infectious sign (core body temperature $>38^{\circ} \mathrm{C}$, shivering, white blood cell count $>10$ or $<4$ cells $\times 10^{9} \mathrm{~L}^{-1}$ ) or one finding during auscultation (rales or crepitation). In all patients with CAP at that time an infiltrate was radiologically confirmed. Patients with language restriction or dementia that precluded from providing written informed consent, intravenous drug use, terminal condition or hospital-acquired pneumonia were excluded. Participants were allowed to receive corticosteroids or short-term antibiotic pretreatment but were excluded if they suffered from severe immunosuppression or received long-term antibiotic pretreatment at ED presentation.

The Ethics Committee of the University of Basel approved the initial study protocol and all patients provided written informed consent for the first trial including consent for the use of their data in secondary biomarker analyses and follow-up. The ProHOSP trial was registered at http://controlled-trials.com (identifier ISRCTN95122877).

\section{Analysis of blood biomarker}

Blood samples from all patients were collected upon ED admission. They were prepared to plasma and stored frozen at $-80^{\circ} \mathrm{C}$ for later measurement of different blood markers.

The initial cohort consisted of 925 CAP patients. Baseline TRP, 5-HT, and KYN levels were measured in the plasma samples of 279 randomly selected subjects with available plasma samples. Therefore LC-MS/MS analysis was performed using an Ultimate 3000 UHPLC (Thermo Fisher, San Jose, USA) system coupled to an ABSciex 5500 quadrupole mass spectrometer (ABSciex, Darmstadt, Germany) and AbsoluteIDQ p180 Kit (BIOCRATES Life Sciences AG, Innsbruck, Austria) as described in previous studies [16-18]. Briefly, a targeted profiling scheme is used to screen quantitatively for known small molecule metabolites using multiple reaction monitoring, neutral loss, and precursor ion scans. Quantification of the metabolites of the biological sample is achieved by reference to appropriate internal standards. Concentrations of all analyzed metabolites are reported in $\mu \mathrm{mol} / \mathrm{L}$ [18]. Eleven measurements with a value of $0 \mu \mathrm{mol} / \mathrm{L}$ were excluded from our analysis because they were considered to be erroneous measurements. Based on the literature, normal range of TRP is at least $20 \mu \mathrm{mol} / \mathrm{L}$ or more [19]. Finally, we had a total number of $268 \mathrm{TRP}, 250 \mathrm{KYN}$, and 213 5-HT measurements for statistical analysis.

In infection, a decrease of TRP and an increase of KYN at the same time was previously found $[20,21]$. Thus, the ratio of KYN/ TRP was proposed to be a sensitive indicator of IDO activity and is widely used in current research [22]. The same principle was done for estimation of TPH. 


\section{Main outcome measurements}

The primary endpoint was adverse outcome including 30-days allcause mortality or ICU admission. As secondary endpoints, mortality at 30 days and 6-year mortality were defined. Survival status has been confirmed by structured phone interviews through trained medical students at day 30, 180 and 540 and at 6 years [15, 23]. The pneumonia severity index (PSI) and quick sepsis-related organ failure assessment: altered mental status, fast respiratory rate ( $\geq 22$ breaths per min), low blood pressure (systolic blood pressure $\leq 100 \mathrm{mmHg}$ ) (qSOFA) were used as first clinical assessment scores. PSI is composited by sex, past medical history, clinical, laboratory and radiological findings [24]. Altered mental status, fast respiratory rate ( $\geq 22$ breaths per min) and low blood pressure (systolic blood pressure $\leq 100 \mathrm{mmHg}$ ) are used for qSOFA [25].

\section{Statistical analysis}

All statistical analysis were performed using STATA 12.1 (Stata Corp, College Station, TX, USA) and $\mathrm{p}<0.05$ (two-tailed) was considered to indicate statistical significance. Unless stated otherwise, categorical variables are expressed as percentages (numbers) and continuous variables as medians [interquartile range (IQR)]. The distribution of the biomarkers TRP, 5-HT, and KYN were skewed. After logarithmic transformation with a base of 10 , the distribution of the biomarker data approximated a normal distribution. First, we calculated Spearman rank correlation to investigate effects of the metabolites of the TRP/5-HT pathway and inflammation [assessed by C-reactive protein (CRP)], infection (assessed by PCT), sepsis severity (assessed by qSOFA) and CAP severity (assessed by PSI). Next, we used univariate and multivariate logistic regression models to examine the association of the metabolites and clinical outcomes, namely adverse outcome defined as death and/or ICU admission, as well as death, both within 30 days of follow-up. The multivariate models were adjusted for age, gender, and comorbidities. Also, univariate and multivariate Cox regression models were used to investigate associations between metabolites levels at baseline and all-cause mortality over 6 years. The area under curve (AUC) with 95\% CI (confidence interval) is presented to illustrate discrimination and predictive power. KaplanMeier curves were used to represent mortality based on quartiles of the biomarkers (highest vs. lower three, except TRP lowest vs. higher three).

\section{Results}

\section{Patient characteristics}

Baseline characteristics of patients stratified by adverse outcome are presented in Table 1 . In general, patients with an adverse outcome were older and had a trend for a higher body mass index. There was a high burden of comorbidities with chronic kidney disease being more frequent in patients with an adverse outcome. Also, patients with an adverse clinical outcome had higher PSI and qSOFA-scores.

When looking at different metabolites of the TRP/5-HT pathway, we found a negative correlation of IDO and TRP (Spearman rank: $\mathrm{r}^{2}=-0.34, \mathrm{p}<0.001$ ), as well as a positive correlation of IDO and KYN (Spearman rank: $\mathrm{r}^{2}=0.69$, $\mathrm{p}<0.001$ ) suggesting that higher IDO activity correlates with the catabolism of TRP to KYN.

\section{Association of the tryptophan/serotonin pathway in inflammation and infection}

In a first step, we investigated the association of the metabolites of the TRP/5-HT pathway with inflammation (assessed by CRP), infection (assessed by PCT), sepsis severity (assessed by qSOFA) and CAP severity (assessed by PSI). We found positive correlations of IDO with all of the markers above, particularly regarding PCT (Spearman rank: $\left.\mathrm{r}^{2}=0.34, \mathrm{p}<0.001\right)$ and PSI (Spearman rank: $\mathrm{r}^{2}=0.39$, $\mathrm{p}<0.001$ ) (see Table 2). Also, TRP was negatively correlated with CRP (Spearman rank: $\mathrm{r}^{2}=-0.24, \mathrm{p}<0.001$ ) and PCT (Spearman rank: $\left.\mathrm{r}^{2}=-0.24, \mathrm{p}<0.001\right)$. KYN showed a positive association with qSOFA (Spearman rank: $\mathrm{r}^{2}=0.14$, $\mathrm{p}<0.05$ ) and PSI (Spearman rank: $\mathrm{r}^{2}=0.34, \mathrm{p}<0.001$ ).

\section{Association of the tryptophan/serotonin pathway and adverse outcome}

Next, we investigated the metabolites of the TRP/5-HT pathway with clinical outcomes. As illustrated in Figure 2, TRP was lower (median $41.2 \mu \mathrm{mol} / \mathrm{L}$ vs. $52.2 \mu \mathrm{mol} / \mathrm{L}$, $p=0.041$ ) and the ratio of IDO was increased (median 101.2 vs. $67, \mathrm{p}=0.002$ ) in patients with, as compared to those without an adverse outcome. This resulted in a trend of higher levels of KYN (median $4.3 \mu \mathrm{mol} / \mathrm{L}$ vs. $3.1 \mu \mathrm{mol} / \mathrm{L}$, $\mathrm{p}=0.088$ ). There were no significant differences in 5 -HT concentrations.

These results were also confirmed in Kaplan-Meier curves based on the highest quartile compared to the three lower quartiles to illustrate the association of the biomarkers with an adverse clinical outcome at 30 days (Figure 3). Because of the inverse association of TRP, we compared the lowest quartile with the three higher quartiles for TRP plasma levels. The strongest effect was seen for TRP ( $p \log$ rank $=0.005)$ and IDO ( $p \log$ rank $=0.011)$. No effect was found for 5-HT. 
Table 1: Baseline characteristics of the study population by $1^{\circ}$ outcome.

\begin{tabular}{|c|c|c|c|}
\hline Characteristics & $\begin{array}{r}\text { No adverse outcome } \\
n=240\end{array}$ & $\begin{array}{r}\text { Adverse outcome } \\
n=28\end{array}$ & p-Value \\
\hline \multicolumn{4}{|l|}{ Demographic characteristics } \\
\hline Age median, years & $71(57,82)$ & $76(66.5,82.5)$ & 0.120 \\
\hline Male & $144(60.0 \%)$ & $17(60.7 \%)$ & 0.940 \\
\hline BMI & $24.7(22.0,27.7)$ & $26.6(24.4,29.5)$ & 0.071 \\
\hline \multicolumn{4}{|l|}{ Comorbidities } \\
\hline Coronary artery disease & $43(17.9 \%)$ & $8(28.6 \%)$ & 0.170 \\
\hline Congestive heart failure & $31(12.9 \%)$ & $4(14.3 \%)$ & 0.840 \\
\hline Chronic kidney disease & $46(19.2 \%)$ & $15(53.6 \%)$ & $<0.001$ \\
\hline Diabetes mellitus & 35 (14.6\%) & $5(17.9 \%)$ & 0.650 \\
\hline Neoplastic disease & $26(10.8 \%)$ & $5(17.9 \%)$ & 0.270 \\
\hline Pneumopathy & $58(24.2 \%)$ & $11(39.3 \%)$ & 0.083 \\
\hline \multicolumn{4}{|l|}{ Initial clinical findings } \\
\hline Pulse rate per minute & $94(82,106)$ & $97(79,114)$ & 0.910 \\
\hline Temperature, ${ }^{\circ} \mathrm{C}$ & $38(37.3,39)$ & $37.4(36.7,38.4)$ & 0.022 \\
\hline Systolic BP, mmHg & $131(120,146.5)$ & $125.5(103.5,139.5)$ & 0.081 \\
\hline \multicolumn{4}{|l|}{ Initial clinical risk assesment } \\
\hline \multicolumn{4}{|l|}{ PSI } \\
\hline Class 1-2 & $67(27.9 \%)$ & $3(10.7 \%)$ & 0.001 \\
\hline Class 3 & $55(22.9 \%)$ & $1(3.6 \%)$ & \\
\hline Class $4-5$ & $118(49.2 \%)$ & $24(85.7 \%)$ & \\
\hline \multicolumn{4}{|l|}{ CURB-65 } \\
\hline 0-1 point & $114(47.5 \%)$ & $5(17.9 \%)$ & $<0.001$ \\
\hline $2-3$ points & $120(50.0 \%)$ & $16(57.1 \%)$ & \\
\hline $4-5$ points & $6(2.5 \%)$ & $7(25.0 \%)$ & \\
\hline \multicolumn{4}{|l|}{ qSOFA } \\
\hline 0 point & $128(53.3 \%)$ & $5(17.9 \%)$ & $<0.001$ \\
\hline 1 point & $99(41.2 \%)$ & $16(57.1 \%)$ & \\
\hline $2-4$ points & $13(5.4 \%)$ & $7(25.0 \%)$ & \\
\hline \multicolumn{4}{|l|}{ Inflammatory markers } \\
\hline $\mathrm{CRP}, \mathrm{mg} / \mathrm{L}$ & $127.5(61.2,236.5)$ & $208.0(90.5,360.0)$ & 0.022 \\
\hline $\mathrm{PCT}, \mu \mathrm{g} / \mathrm{L}$ & $0.38(0.14,2.88)$ & $0.65(0.21,6.69)$ & 0.089 \\
\hline Creatinine, $\mu \mathrm{mol} / \mathrm{L}$ & $85(69,108)$ & $117(73,179)$ & 0.007 \\
\hline Art. pH & $7.45(7.43,7.49)$ & $7.44(7.38,7.47)$ & 0.029 \\
\hline \multicolumn{4}{|l|}{ Tryptophan/serotonin metabolism } \\
\hline Tryptophan, $\mu \mathrm{mol} / \mathrm{L}$ & $52.2(40.3,75.3)$ & $41.2(31.9,66.4)$ & 0.041 \\
\hline Serotonin, $\mu \mathrm{mol} / \mathrm{L}$ & $0.2(0.1,0.3)$ & $0.2(0.1,0.2)$ & 0.940 \\
\hline Kynurenine, $\mu \mathrm{mol} / \mathrm{L}$ & $3.1(1.7,5.6)$ & $4.3(1.7,9.2)$ & 0.230 \\
\hline Tryptophan hydroxylase ratio & $3.5(2.2,5.7)$ & $4.1(2.6,8.4)$ & 0.088 \\
\hline Indoleamine 2,3-dioxygenase ratio & $67.0(44.5,99.3)$ & $101.2(61.5,186.3)$ & 0.002 \\
\hline
\end{tabular}

Data are presented as median (IQR) or no. (\%). Bold $p$-values are statistically significant at $p<0.05$. IQR, interquartile range (25th-75th percentiles). Comorbidities were identified based on medical records of general practitioners, patient report, or both. Adverse outcome, 30-days all-cause mortality or ICU admission. PSI, pneumonia severity index; CURB-65, pneumonia risk scoring system; qSOFA, quick sepsis-related organ failure assessment; CRP, C-reactive protein; PCT, procalcitonin; Art. pH, arterial pH.

\section{Association between biomarkers and short- and long-term follow-up}

Further, we also calculated univariate and multivariate regression analysis to investigate the association of metabolites of the TRP/5-HT pathway and short-term outcome (death and adverse outcome within 30 days) as well as long-term outcome (mortality within 6 years). As presented in Table 3, TRP showed again an inverse association with an adverse short-term outcome [adjusted odds ratio (OR) 0.11 (95\% CI 0.02-0.7), $\mathrm{p}=0.021$, but was not associated with long-term outcome. KYN was associated with short- and long-term outcome in univariate analysis. After multivariate adjustment, these associations were no longer significant. Similarly, IDO showed a significant association in univariate analysis, but in multivariate 
Table 2: Association of tryptophan/serotonin pathway with inflammation, infection, severity and sepsis score.

\begin{tabular}{lllll}
\hline & CRP (inflammation) & PCT (infection) & PSI (severity) & qSOFA (sepsis) \\
\hline Tryptophan & $-\mathbf{0 . 2 4}(\mathrm{p}=\mathbf{0 . 0 0 1 )}$ & $-\mathbf{0 . 2 4}(\mathrm{p}=\mathbf{0 . 0 0 1 )}$ & $-0.05(\mathrm{p}=0.479)$ & $-0.02(\mathrm{p}=0.778)$ \\
Serotonin & $0.01(\mathrm{p}=0.891)$ & $-0.10(\mathrm{p}=0.138)$ & $-\mathbf{0 . 1 9}(\mathrm{p}=\mathbf{0 . 0 0 6 )}$ & $-0.02(\mathrm{p}=0.753)$ \\
Kynurenine & $-0.08(\mathrm{p}=0.251)$ & $0.14(\mathrm{p}=0.054)$ & $\mathbf{0 . 3 4}(\mathrm{p}<\mathbf{0 . 0 0 1 )}$ & $0.14(\mathrm{p}=0.046)$ \\
$\begin{array}{l}\text { Tryptophan } \\
\text { hydroxylase }\end{array}$ & $\mathbf{0 . 1 4}(\mathrm{p}=\mathbf{0 . 0 4 6 )}$ & $0.04(\mathrm{p}=0.555)$ & $-\mathbf{0 . 1 7}(\mathrm{p}=\mathbf{0 . 0 1 5 )}$ & $-0.03(\mathrm{p}=0.663)$ \\
$\begin{array}{l}\text { Indoleamine } \\
\text { 2,3-dioxygenase }\end{array}$ & $\mathbf{0 . 1 4}(\mathrm{p}=\mathbf{0 . 0 4 2 )}$ & $\mathbf{0 . 3 4}(\mathrm{p}<\mathbf{0 . 0 0 1 )}$ & $\mathbf{0 . 3 9}(\mathrm{p}<\mathbf{0 . 0 0 1 )}$ & $\mathbf{0 . 2 0}(\mathrm{p}=\mathbf{0 . 0 0 4 )}$ \\
\hline
\end{tabular}

Data for Spearman rank analysis are given as $\mathrm{r}^{2}$. Bold $\mathrm{p}$-values are statistically significant at $\mathrm{p}<0.05$. CRP, C-reactive protein; $\mathrm{PCT}$, procalcitonin; PSI, pneumonia severity index; qSOFA, quick sepsis-related organ failure assessment.
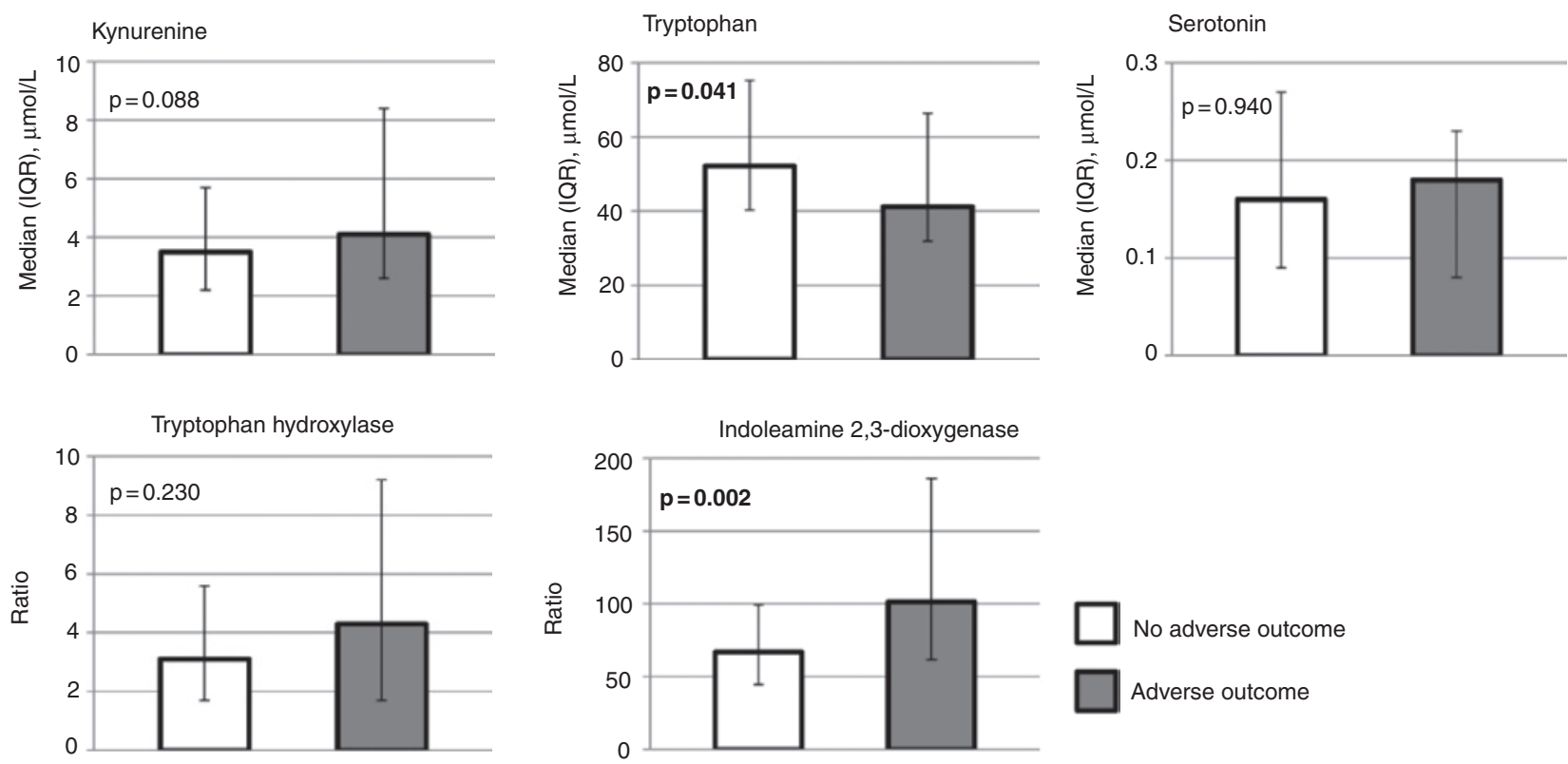

No adverse outcome

Adverse outcome

Figure 2: Medians and their interquartile range (IQR) by $1^{\circ}$ outcome.

Bold $p$-values are statistically significant at $p<0.05$.

analysis only short-term outcome remained significant.

No associations were found for 5-HT.

\section{Association of IDO with comorbidities}

Finally, we conducted linear regression models to find out which comorbidities and parameters were associated with higher marker levels focusing on IDO, KYN, and TRP (Table 4). The strongest factor thereby was chronic kidney disease for IDO [adjusted correlation coefficient 0.27 (95\% CI 0.2-0.4), $\mathrm{p}<0.001]$, TRP [0.12 (95\% CI - 0.2-0), $\mathrm{p}=0.004]$, and KYN [ $0.12(95 \% \mathrm{CI}-0.2-0), \mathrm{p}=0.004]$, as well as age for TRP [0.02 (95\% CI 0-0), $\mathrm{p}=0.032]$ and KYN [0.03 (95\% CI 0-0.1), $\mathrm{p}=0.016]$.

\section{Discussion}

This study, examining the performance of metabolites of the TRP/5-HT pathway in a well-characterized cohort of CAP patients followed for over 6 years, revealed three key findings on hospital admission. First, we found a strong activation of IDO leading to a shift in metabolism from TRP to KYN dependent on infection, inflammation, and severity. No changes were found for circulating 5-HT levels. Second, higher levels of IDO and KYN, as well as lower levels of TRP were associated with clinical patient outcomes. These associations, however, were partly explained by age and comorbidities as effects in multivariate models were less impressive. Third, 

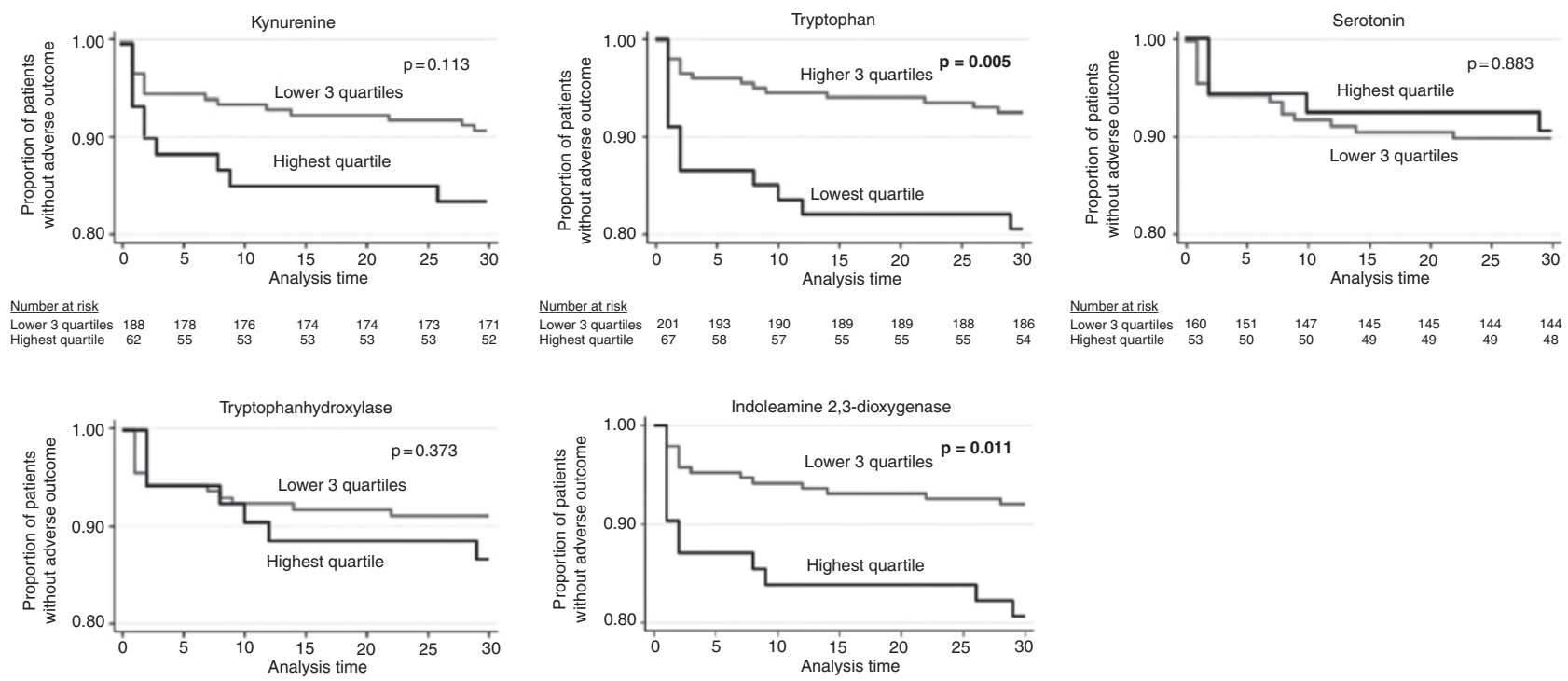

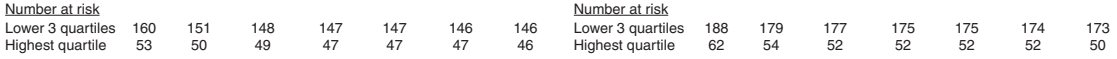

Figure 3: Kaplan-Meier curves by adverse outcome.

Bold $p$-values are statistically significant at $p<0.05$. Adverse outcome, 30-days all-cause mortality or ICU admission.

Table 3: Association of tryptophan/serotonin pathway by short-/long-term follow-up.

\begin{tabular}{|c|c|c|c|c|}
\hline & \multicolumn{2}{|l|}{ OR $(95 \% \mathrm{Cl}), \mathrm{p}$-Value } & & \multirow{2}{*}{$\frac{\mathrm{HR}(95 \% \mathrm{Cl}), \mathrm{p} \text {-Value }}{\text { Death } 6 \text { years }}$} \\
\hline & Adverse outcome & Death 30 days & & \\
\hline \multicolumn{5}{|l|}{ Tryptophan } \\
\hline Univariate $O R$ & $0.29(0.09$ to 1.2$), p=0.089$ & $0.34(0.05$ to 2.5$), p=0.293$ & Univariate HR & $1.17(0.6$ to 2.5$), p=0.684$ \\
\hline Multivariate $\mathrm{OR}^{\mathrm{a}}$ & $0.11(0.02$ to 0.7$), p=0.021$ & $0.16(0.01$ to 3.4$), p=0.240$ & Multivariate $\mathrm{HR}^{\mathrm{a}}$ & $1.19(0.5$ to 3.0$), p=0.702$ \\
\hline AUC & $0.38(0.3$ to 0.5$)$ & $0.44(0.3$ to 0.6$)$ & AUC & $0.52(0.4$ to 0.6$)$ \\
\hline \multicolumn{5}{|l|}{ Serotonin } \\
\hline Univariate $\mathrm{OR}$ & 1.00 (0.3 to 2.9$), p=0.993$ & 0.95 (0.2 to 3.9), $p=0.941$ & Univariate HR & $0.91(0.6$ to 1.4$), p=0.672$ \\
\hline Multivariate $\mathrm{OR}^{\mathrm{a}}$ & $1.59(0.4$ to 6.3$), p=0.508$ & $1.42(0.2$ to 8.4$), p=0.703$ & Multivariate $\mathrm{HR}^{\mathrm{a}}$ & $1.20(0.7$ to 2.1$), p=0.515$ \\
\hline AUC & $0.50(0.4$ to 0.6$)$ & $0.52(0.3$ to 0.7$)$ & AUC & $0.46(0.4$ to 0.5$)$ \\
\hline \multicolumn{5}{|l|}{ Kynurenine } \\
\hline Univariate $\mathrm{OR}$ & 4.54 (1.1 to 18.2$), p=0.033$ & 10.1 (1.3 to 79.2$), p=0.028$ & Univariate HR & $1.96(1.0$ to 3.7$), p=0.036$ \\
\hline Multivariate $\mathrm{OR}^{\mathrm{a}}$ & 1.81 ( 0.3 to 9.6$), p=0.485$ & 4.23 ( 0.2 to 93.2$), p=0.361$ & Multivariate $\mathrm{HR}^{\mathrm{a}}$ & $0.86(0.4$ to 1.8$), p=0.676$ \\
\hline AUC & $0.60(0.5$ to 0.7$)$ & $0.68(0.5$ to 0.8$)$ & AUC & $0.58(0.5$ to 0.7$)$ \\
\hline \multicolumn{5}{|c|}{ Tryptophan hydroxylase } \\
\hline Univariate $O R$ & $2.20(0.7$ to 6.5$), p=0.154$ & $1.50(0.4$ to 6.1$), p=0.571$ & Univariate HR & $0.92(0.6$ to 1.4$), p=0.710$ \\
\hline Multivariate $\mathrm{OR}^{\mathrm{a}}$ & $5.16(1.1$ to 24.0$), p=0.037$ & $2.27(0.4$ to 14$), p=0.376$ & Multivariate $\mathrm{HR}^{\mathrm{a}}$ & $1.13(0.7$ to 1.9$), p=0.653$ \\
\hline AUC & $0.58(0.4$ to 0.7$)$ & $0.54(0.3$ to 0.7$)$ & AUC & $0.45(0.4$ to 0.5$)$ \\
\hline \multicolumn{5}{|c|}{ Indoleamine 2,3-dioxygenase } \\
\hline Univariate OR & $11.2(2.7$ to 46.5$), p=0.001$ & 12.37 ( 1.8 to 85.9 ), $p=0.011$ & Univariate HR & 1.90 (1.0 to 3.5), $p=0.040$ \\
\hline Multivariate $O^{a}$ & 9.10 (1.4 to 59.5$), p=0.021$ & $5.18(0.3$ to 105.6$), p=0.284$ & Multivariate $\mathrm{HR}^{\mathrm{a}}$ & 0.77 ( 0.3 to 1.7$), p=0.526$ \\
\hline AUC & $0.69(0.6$ to 0.8$)$ & $0.69(0.5$ to 0.9$)$ & AUC & $0.60(0.5$ to 0.7$)$ \\
\hline
\end{tabular}

Data for AUC, univariate and multivariate linear or Cox regression analyses are given as coefficient $(95 \% \mathrm{Cl})$. Bold p-values are statistically significant at $\mathrm{p}<0.05$. $\mathrm{Cl}$, confidence interval; OR, odds ratio; HR, hazard ratio. Adverse outcome, 30-days all-cause mortality or ICU admission. a Multivariate model: adjusted for patient age, gender and comorbidities (coronary artery disease, congestive heart failure, chronic kidney disease, diabetes mellitus, neoplastic disease, pneumopathy, body mass index).

the main parameters that influence the metabolites of the TRP/5-HT pathway were chronic kidney disease and age.
The present study showed a decrease of TRP in patients with CAP. This decrease correlated with higher disease severity as measured by higher levels of PCT 
Table 4: Association with demographic characteristics and comorbidities in multivariate analyses.

\begin{tabular}{|c|c|c|c|}
\hline & \multicolumn{3}{|l|}{ OR $(95 \% \mathrm{Cl}), \mathrm{p}$-Value } \\
\hline & Tryptophan & Kynurenine & Indoleamine 2,3-dioxygenase \\
\hline \multicolumn{4}{|l|}{ Patient characteristics } \\
\hline Age decades & $0.02(0$ to 0$), p=0.032$ & $0.03(0$ to 0.1$), p=0.016$ & $0.02(-0.01$ to 0.04$), p=0.180$ \\
\hline Male & $-0.06(-0.1$ to 0$), p=0.080$ & $-0.06(-0.1$ to 0$), p=0.190$ & $0.03(-0.1$ to 0.1$), p=0.535$ \\
\hline \multicolumn{4}{|l|}{ Comorbidities } \\
\hline Coronary artery disease & $-0.05(-0.1$ to 0$), p=0.263$ & $-0.03(-0.1$ to 0.1$), p=0.563$ & $0.004(-0.1$ to 0.1$), p=0.936$ \\
\hline Congestive heart failure & $-0.02(-0.1$ to 0.1$), p=0.756$ & $0.001(-0.1$ to 0.1$), p=0.982$ & $-0.02(-0.1$ to 0.1$), p=0.806$ \\
\hline Chronic kidney disease & $-0.12(-0.2$ to 0$), p=0.004$ & $0.15(0$ to 0.3$), p=0.007$ & $0.27(0.2$ to 0.4$), p<0.001$ \\
\hline Diabetes mellitus & $0.01(-0.1$ to 0.1$), p=0.807$ & $0.01(-0.1$ to 0.1$), p=0.916$ & $0.01(-0.1$ to 0.1$), p=0.861$ \\
\hline Pneumopathy & $0.005(-0.1$ to 0.1$), p=0.898$ & $0.01(-0.1$ to 0.1$), p=0.858$ & $0.02(-0.1$ to 0.1$), p=0.618$ \\
\hline Neoplastic disease & $-0.09(-0.2$ to 0$), p=0.083$ & $-0.01(-0.1$ to 0.1$), p=0.884$ & $0.07(-0.1$ to 0.2$), p=0.258$ \\
\hline Body mass index & $0.004(0$ to 0$), p=0.164$ & $0.004(0$ to 0$), p=0.257$ & $0.004(-0.01$ to 0.01$), p=0.983$ \\
\hline
\end{tabular}

Data for multivariate linear regression analyses are given as coefficient $(95 \% \mathrm{Cl})$. Bold $\mathrm{p}$-values are statistically significant at $\mathrm{p}<0.05$. $\mathrm{Cl}$, confidence interval.

and CRP, and higher risk scores (qSOFA and PSI). These results are in line with previous research [26-28]. The physiopathological mechanism of decreasing TRP during infection and inflammation is a matter of current discussion [29]. It has been hypothesized early on that diseaseassociated starvation and depletion by microbes may cause these effects. More recently, it has been proposed that the activation of the TRP/5-HT pathway is part of the host defense against infection and, thus, a beneficial adaptation [30]. These considerations are important because IDO can be therapeutically influenced, which in turn may impact the treatment and recovery of the infection. Recently, a particular substance that inhibits IDO has been developed and tested in animal models, namely the IDO inhibitor 1-methyl-tryptophan [31]. In a mouse model, IDO inhibitors potentiated cancer chemotherapy because of an immune-modulating effect of IDO [32]. Also, a blockade of IDO showed a reduction in mortality from peritonitis and sepsis in mice [27]. Despite these promising early results, clinical data is currently lacking demonstrating whether an IDO inhibitor could improve patient outcome in acute infection and sepsis. In this context, our clinical study showing a severity-dependent activation of IDO and a strong relationship to clinical outcome is relevant and may help to direct future interventional trials to answer the question, whether neutralization of the immunosuppressive effects could lead to a better infection control. Alternatively, an overwhelming reaction due to a suppression of the immune response in the human body would be possible.

Only a few studies have investigated the impact of the TRP/5-HT metabolism in patients with CAP. Similarly to our study, Suzuki et al. [11] found an increase in IDO according to severity in a smaller cohort of CAP patients
(129 subjects). Also, they studied short-term outcome and did multivariate regression analysis with laboratory parameters (e.g. CRP, blood urea nitrogen). The study, however, did not investigate long-term outcome, correlation with comorbidities and 5-HT was not assessed. Our results thus validate these findings, but also expand the results regarding metabolites and clinical significance.

We did not find an increase in 5-HT in patients according to severity and outcome. 5-HT is stored in platelets which are activated during infections resulting potentially in endothelial hyperpermeability [33]. This secretion depends on the 5-HT transporter (SERT) [34]. From our results, it remains unclear why there was no evidence for increased 5-HT levels according to the severity of an infection. Possibly, inhibition by SERT-inhibitors could lower the peripheral levels of 5-HT [3]. Alternatively, pre-analytical issues and low metabolite stability could explain the lack of association.

Further, we investigated factors associated with the activation of the TRP/5-HT pathway. In multivariate analysis, chronic kidney disease was found to be an important predictor for IDO and KYN elevation. This is also in line with previous studies reporting decreased TRP and increased KYN and IDO levels in chronic kidney disease [35-37]. Renal excretion is known to be the main route of KYN elimination [38]. As mentioned above, IDO levels were still associated with adverse events after adjustment for chronic kidney disease, indicating that elevated KYN levels are not attributable to renal excretory impairment alone, but also to up-regulated metabolism (e.g. systemic inflammation). It is evident, that chronic Inflammation is a significant burden to patients with chronic kidney disease and is elevated by disease progression [39]. As suggested by previous clinical trials, elevated IDO and 
KYN levels in chronic kidney diseases possibly indicate a chronic activation of the immune system [40].

As a second factor, age was found to be associated with TRP and KYN. These findings are in line with current research and are possibly explained by chronic inflammation [41, 42].

The main strengths of this study include (i) the wellcharacterized cohort of patients with CAP of different severities representative for patients usually treated in EDs and hospitals, (ii) the long and rigorous follow-up over a period of 6 years, and (iii) all-cause mortality as a primary outcome. Nevertheless, our study has limitations. First, this study was conducted in several hospitals in Switzerland, and results may not be generalizable to other countries. Second, autopsies were not performed to verify the causes of death and therefore this study focused on all-cause mortality rather than acute infection caused events. Third, this was an observational study and as such just hypothesis generating. Finally, the ratios of IDO and TPH are estimated and not measured. In future evaluations, complete measurements of all involved metabolites should be performed including plasma free TRP, determinants of TRP binding (albumin and nonesterified fatty acids), total TRP, TDO, IDO and levels of KYN metabolites [43].

\section{Conclusions}

In hospitalized CAP patients, higher IDO activity and lower TRP levels independently predicted disease severity and short-term adverse outcome. Whether therapeutic modulation of IDO has positive effects on outcome needs further investigation.

Acknowledgments: We are thankful for all patients, patients' relatives and all local general practitioners who participated in this study. In particular, we thank the emergency department, medical clinic, and central laboratory staff of the University Hospital Basel and the Cantonal Hospitals Aarau, Liestal, Lucerne, and Muensterlingen, and the 'Buergerspital' Solothurn for their assistance and technical support. Finally, we acknowledge our ProHOSP Study Group for their important support.

Author contributions: All the authors have accepted responsibility for the entire content of this submitted manuscript and approved submission. PS, MC-C, and BM created the concept and design, wrote the protocol and initiated the initial ProHOSP study. MM and PS drafted the present manuscript and performed the statistical analyses. CS, AH, and LB performed laboratory measurements of p180-Kit. All authors contributed to the data acquisition, interpretation and drafting of the analyses, critical review for important content, and final approval of the manuscript. PS had full access to all data and takes responsibility for the accuracy of the data analysis and the integrity of the work.

The ProHOSP Study Group included: U. Schild, K. Regez, R. Bossart, C. Blum, M. Wolbers, S. Neidert, I. Suter, H.C. Bucher, F. Mueller, A. Chaudry, J. Haeuptle, R. Zarbosky, R. Fiumefreddo, M. Wieland, C. Nussbaumer, A. Christ, R. Bingisser, and K. Schneider (University Hospital Basel, Basel, Switzerland); T. Bregenzer, D. Conen, A. Huber, and J. Staehelin (Kantonsspital Aarau, Aarau, Switzerland); W. Zimmerli, C. Falconnier, and C. Bruehl-hardt (Kantonsspital Baselland, Liestal, Switzerland); C. Henzen and V. Briner (Kantonsspital Luzern, Luzern, Switzerland); T. Fricker, C. Hoess, M. Krause, I. Lambinon, and M. Zueger (Kantonsspital Muensterlingen, Muensterlingen, Switzerland); and R. Thomann, R. Schoenenberger, and R. Luginbuehl (Buergerspital Solothurn, Solothurn, Switzerland).

Research funding: This study was supported in part by the Swiss National Science Foundation (SNSF Professorship, PP00P3_150531/1) and the Research Council of the Kantonsspital Aarau (1410.000.044). The initial trial was funded by the Swiss National Science Foundation (grant SNF 3200BO-116177/1), Santé Suisse, the Gottfried and Julia Bangerter-Rhyner Foundation and BRAHMS Biomarkers.

Employment or leadership: None declared.

Honorarium: None declared.

Competing interests: The funding organization(s) played no role in the study design; in the collection, analysis, and interpretation of data; in the writing of the report; or in the decision to submit the report for publication.

\section{References}

1. Cevikkalp SA, Loker GB, Yaman M, Amoutzopoulos B. A simplified HPLC method for determination of tryptophan in some cereals and legumes. Food Chem 2016;193:26-9.

2. Yeung AW, Terentis AC, King NJ, Thomas SR. Role of indoleamine 2,3-dioxygenase in health and disease. Clin Sci (Lond) 2015;129:601-72.

3. Li Y, Hadden C, Cooper A, Ahmed A, Wu H, Lupashin VV, et al. Sepsis-induced elevation in plasma serotonin facilitates endothelial hyperpermeability. Sci Rep 2016;6:22747.

4. Gal EM, Sherman AD. L-kynurenine: its synthesis and possible regulatory function in brain. Neurochem Res 1980;5:223-39.

5. Carlin JM, Borden EC, Sondel PM, Byrne GI. Biologic-responsemodifier-induced indoleamine 2,3-dioxygenase activity in human peripheral blood mononuclear cell cultures. J Immunol 1987;139:2414-8. 
6. Hayaishi O. Properties and function of indoleamine 2,3-dioxygenase. J Biochem 1976;79:13P-21P.

7. de Stoppelaar SF, van 't Veer C, Claushuis TA, Albersen BJ, Roelofs JJ, van der Poll T. Thrombocytopenia impairs host defense in gram-negative pneumonia-derived sepsis in mice. Blood 2014;124:3781-90.

8. Kochanek KD, Murphy SL, Xu J, Tejada-Vera B. Deaths: final data for 2014. Natl Vital Stat Rep 2016;65:1-122.

9. Brown PD, Lerner SA. Community-acquired pneumonia. Lancet 1998;352:1295-302.

10. File TM. Community-acquired pneumonia. Lancet $2003 ; 362$ : 1991-2001.

11. Suzuki Y, Suda T, Yokomura K, Suzuki M, Fujie M, Furuhashi K, et al. Serum activity of indoleamine 2,3-dioxygenase predicts prognosis of community-acquired pneumonia. J Infect 2011;63:215-22.

12. Mehraj V, Routy JP. Tryptophan catabolism in chronic viral infections: handling uninvited guests. Int J Tryptophan Res 2015;8:41-8.

13. Schuetz P, Christ-Crain M, Wolbers M, Schild U, Thomann R, Falconnier $C$, et al. Procalcitonin guided antibiotic therapy and hospitalization in patients with lower respiratory tract infections: a prospective, multicenter, randomized controlled trial. BMC Health Serv Res 2007;7:102.

14. Schuetz P, Christ-Crain M, Thomann R, Falconnier C, Wolbers $M$, Widmer I, et al. Effect of procalcitonin-based guidelines vs standard guidelines on antibiotic use in lower respiratory tract infections: the ProHOSP randomized controlled trial. J Am Med Assoc 2009;302:1059-66.

15. Alan M, Grolimund E, Kutz A, Christ-Crain M, Thomann R, Falconnier $C$, et al. Clinical risk scores and blood biomarkers as predictors of long-term outcome in patients with community-acquired pneumonia: a 6-year prospective follow-up study. J Intern Med 2015;278:174-84.

16. Weinberger KM. Metabolomics in diagnosing metabolic diseases. Therapeutische Umschau Revue therapeutique 2008;65:487-91.

17. Yet I, Menni C, Shin SY, Mangino M, Soranzo N, Adamski J, et al. Genetic influences on metabolite levels: a comparison across metabolomic platforms. PLoS One 2016;11:e0153672.

18. Illig T, Gieger C, Zhai G, Romisch-Margl W, Wang-Sattler R, Prehn $C$, et al. A genome-wide perspective of genetic variation in human metabolism. Nat Genet 2010;42:137-41.

19. Lepage N, McDonald N, Dallaire L, Lambert M. Age-specific distribution of plasma amino acid concentrations in a healthy pediatric population. Clin Chem 1997;43:2397-402.

20. Byrne GI, Lehmann LK, Landry GJ. Induction of tryptophan catabolism is the mechanism for gamma-interferon-mediated inhibition of intracellular Chlamydia psittaci replication in T24 cells. Infect Immun 1986;53:347-51.

21. Werner ER, Fuchs D, Hausen A, Jaeger H, Reibnegger G, WernerFelmayer $\mathrm{G}$, et al. Tryptophan degradation in patients infected by human immunodeficiency virus. Biol Chem Hoppe Seyler 1988;369:337-40.

22. Widner B, Werner ER, Schennach H, Wachter H, Fuchs D. Simultaneous measurement of serum tryptophan and kynurenine by HPLC. Clin Chem 1997;43:2424-6.

23. Guertler C, Wirz B, Christ-Crain M, Zimmerli W, Mueller B, Schuetz P. Inflammatory responses predict long-term mortality risk in community-acquired pneumonia. Eur Respir J 2011;37:1439-46.

24. Fine MJ, Auble TE, Yealy DM, Hanusa BH, Weissfeld LA, Singer $\mathrm{DE}$, et al. A prediction rule to identify low-risk patients with community-acquired pneumonia. N Engl J Med 1997;336:243-50.

25. Seymour CW, Liu VX, Iwashyna TJ, Brunkhorst FM, Rea TD, Scherag A, et al. Assessment of clinical criteria for sepsis: for the third international consensus definitions for sepsis and septic shock (sepsis-3). J Am Med Assoc 2016;315:762-74.

26. Changsirivathanathamrong D, Wang Y, Rajbhandari D, Maghzal GJ, Mak WM, Woolfe C, et al. Tryptophan metabolism to kynurenine is a potential novel contributor to hypotension in human sepsis. Crit Care Med 2011;39:2678-83.

27. Hoshi M, Osawa Y, Ito H, Ohtaki H, Ando T, Takamatsu M, et al. Blockade of indoleamine 2,3-dioxygenase reduces mortality from peritonitis and sepsis in mice by regulating functions of CD11b+ peritoneal cells. Infect Immun 2014;82:4487-95.

28. Wang Y, Liu H, McKenzie G, Witting PK, Stasch JP, Hahn M, et al. Kynurenine is an endothelium-derived relaxing factor produced during inflammation. Nat Med 2010;16:279-85.

29. Badawy AA. The tryptophan utilization concept in pregnancy. Obstet Gynecol Sci 2014;57:249-59.

30. Bessede A, Gargaro M, Pallotta MT, Matino D, Servillo G, Brunacci C, et al. Aryl hydrocarbon receptor control of a disease tolerance defence pathway. Nature 2014;511:184-90.

31. Muller AJ, DuHadaway JB, Donover PS, Sutanto-Ward E, Prendergast GC. Inhibition of indoleamine 2,3-dioxygenase, an immunoregulatory target of the cancer suppression gene Bin1, potentiates cancer chemotherapy. Nat Med 2005;11:312-9.

32. Muller A, Heseler K, Schmidt SK, Spekker K, Mackenzie CR, Daubener W. The missing link between indoleamine 2,3-dioxygenase mediated antibacterial and immunoregulatory effects. J Cell Mol Med 2009;13:1125-35.

33. Berger M, Gray JA, Roth BL. The expanded biology of serotonin. Annu Rev Med 2009;60:355-66.

34. Majno G, Palade GE, Schoefl GI. Studies on inflammation. II. The site of action of histamine and serotonin along the vascular tree: a topographic study. J Biophys Biochem Cytol 1961;11:607-26.

35. Pawlak D, Tankiewicz A, Matys T, Buczko W. Peripheral distribution of kynurenine metabolites and activity of kynurenine pathway enzymes in renal failure. J Physiol Pharmacol 2003;54:175-89.

36. Saito K, Fujigaki S, Heyes MP, Shibata K, Takemura M, Fujii $\mathrm{H}$, et al. Mechanism of increases in L-kynurenine and quinolinic acid in renal insufficiency. Am J Physiol Renal Physiol 2000;279:F565-72.

37. Schefold JC, Zeden JP, Fotopoulou C, von Haehling S, Pschowski $\mathrm{R}$, Hasper D, et al. Increased indoleamine 2,3-dioxygenase (IDO) activity and elevated serum levels of tryptophan catabolites in patients with chronic kidney disease: a possible link between chronic inflammation and uraemic symptoms. Nephrol Dial Transplant 2009;24:1901-8.

38. Holmes EW. Determination of serum kynurenine and hepatic tryptophan dioxygenase activity by high-performance liquid chromatography. Anal Biochem 1988;172:518-25.

39. Nakanishi I, Moutabarrik A, Okada N, Kitamura E, Hayashi A, Syouji T, et al. Interleukin- 8 in chronic renal failure and dialysis patients. Nephrol Dial Transplant 1994;9:1435-42. 
40. Zinellu A, Sotgia S, Mangoni AA, Sanna M, Satta AE, Carru C. Impact of cholesterol lowering treatment on plasma kynurenine and tryptophan concentrations in chronic kidney disease: relationship with oxidative stress improvement. Nutr Metab Cardiovasc Dis 2015;25:153-9.

41. de Bie J, Guest J, Guillemin GJ, Grant R. Central kynurenine pathway shift with age in women. J Neurochem 2016;136:995-1003.
42. Mangge H, Summers KL, Meinitzer A, Zelzer S, Almer G, Prassl $R$, et al. Obesity-related dysregulation of the tryptophan-kynurenine metabolism: role of age and parameters of the metabolic syndrome. Obesity (Silver Spring) 2014;22:195-201.

43. Badawy AA, Namboodiri AM, Moffett JR. The end of the road for the tryptophan depletion concept in pregnancy and infection. Clin Sci (Lond) 2016;130:1327-33. 